\section{The Increased Risk of Thromboembolic Events in Patients with Rheumatoid Arthritis}

\section{To the Editor:}

We read with great interest the article published in The Journal by Luque Ramos, et $a l^{1}$, in which the comorbidities of rheumatoid arthritis (RA) are detailed, explaining specifically the thromboembolic processes. The authors mentioned that the risk was double in patients with RA compared to controls. We present 2 cases in which the diagnosis of pulmonary thromboembolism was made in patients whose only known risk factor was RA.

It has been widely studied and shown that patients with systemic inflammatory diseases such as RA have a higher risk of mortality from cardiovascular diseases than the healthy population. However, it is postulated that chronic inflammation favors a procoagulant state, which leads to a higher incidence of thromboembolic diseases. This hypothesis has been shown retrospectively, assessing the incidence of both deep venous thrombosis and pulmonary embolism in cohorts affected by RA and comparing them with those of the general population.

The first case is a 45-year-old patient with seropositive RA diagnosed in 2012 and treated with methotrexate at a dose of $17.5 \mathrm{mg}$ and prednisone at a dose of $20 \mathrm{mg}$ a day, without other medical history of interest. The second case is an 86-year-old patient without toxic habits and allergic to vitamin B12, diagnosed with seropositive RA with positive anticyclic citrullinated peptide in 2004 and in usual treatment with prednisolone $20 \mathrm{mg} /$ day, having never received treatment with disease-modifying antirheumatic drugs (DMARD). Both patients were diagnosed with pulmonary thromboembolism, initiating treatment with anticoagulants with a correct subsequent course. In the 2 cases, both the study of hidden neoplasia and the alteration in coagulation factors were negative, ruling out other causes of pulmonary thromboembolism.

RA means a continuous proinflammatory state, although this can be controlled with DMARD. Inflammation of autoimmune diseases modulates thrombotic responses by procoagulant hyperstimulation, decreased anticoagulant processes, and suppression of fibrinolysis.

Patients with RA have a considerably shorter life expectancy than the healthy population, mainly owing to an increase in the incidence of cardiovascular events ${ }^{2,3}$. To date, most studies focus on explaining the relationship between the proinflammatory state and arteriosclerotic alterations and their complications. However, there are few studies of the relationship between the systemic entity and thromboembolic disease [either deep vein thrombosis (DVT) or pulmonary thromboembolism]. In 2012, Zöller, et al showed that hospitalized patients with RA had a higher risk of DVT than the rest of the hospitalized population, especially after the first year when the risk of pulmonary thromboembolism was up to 6 times higher, and then decreased over the next 5 years postdiagnosis ${ }^{4}$. The problem with these studies is that they were performed on a population with an RA exacerbation (the main reason for hospital admission), without assessing thromboembolic risk in the population with nonhospitalized RA (which is the predominant population $)^{5,6}$. In 2014, Choi, et al conducted a study to evaluate the increased risk of DVT and pulmonary thromboembolism in the nonhospitalized population ${ }^{2}$. They demonstrated that patients with RA had a risk ratio (RR) of 2.23 (95\% CI 1.75-2.86) of having pulmonary thromboembolism, compared to the general population, adjusting these results by sex, age, and time of diagnosis of the disease. When adjusting the results for body mass index, smoking, hospitalizations, and other comorbidities (such as chronic obstructive pulmonary disease), the RR remained 2.16 (95\% CI 1.68-2.79) for the pulmonary embolism and 2.16 (95\% CI 1.74-2.69) for venous thromboembolism. The cause of the increased risk in this population, although not thoroughly studied, may be that proinflammatory states are a key factor for the correct function of the endothelium in both arterial and venous circulation ${ }^{7}$.

Patients with RA maintain a chronic proinflammatory state that determines a dysfunction in the vascular endothelium (arterial and venous), thus increasing the risk of thromboembolic events.

RAQUEL ARÀNEGA GONZALEZ ${ }^{\circledR}$, MD, Internal Medicine Department; JORDI JUANOLA PLA, MD, Pulmonology Department; CONCEPCIÓN FALGA TIRADO, MD, Internal Medicine Department; CARLOS LOPERA MARMOL, MD, Internal Medicine Department; PILAR ORTEGA CASTILLO, MD, Pulmonology Department; ANA LAFONT RUZAFA, MD, Rheumatology Department, Consorci Sanitari del Maresme, Mataró, Barcelona, Spain. Address correspondence to Dr. J. Juanola Pla, Pulmonology Department, Carretera de Cirera s/n, Consorci Sanitari del Maresme, Mataró, Barcelona CP 08304, Spain. E-mail: jjuanola@csdm.cat. All patients described signed written informed consent to publish the material.

\section{REFERENCES}

1. Luque Ramos A, Redeker I, Hoffmann F, Callhoff J, Zink A, Albrecht $\mathrm{K}$. Comorbidities in patients with rheumatoid arthritis and their association with patient-reported outcomes: results of claims data linked to questionnaire survey. J Rheumatol 2019;46:564-71.

2. Choi HK, Rho YH, Zhu Y, Cea-Soriano L, Aviña-Zubieta JA, Zhang Y. The risk of pulmonary embolism and deep vein thrombosis in rheumatoid arthritis: a UK population-based outpatient cohort study. Ann Rheum Dis 2013;72:1182-7.

3. Calvo-Alén J, Toloza SM, Fernández M, Bastian HM, Fessler BJ, Roseman JM, et al; LUMINA Study Group. Systemic lupus erythematosus in a multiethnic US cohort (LUMINA). XXV. Smoking, older age, disease activity, lupus anticoagulant, and glucocorticoid dose as risk factors for the occurrence of venous thrombosis in lupus patients. Arthritis Rheum. 2005;52:2060-8.

4. Zöller B, Li X, Sundquist J, Sundquist K. Risk of pulmonary embolism in patients with autoimmune disorders: a nationwide follow-up study from Sweden. Lancet 2012;379:244-9.

5. Ramagopalan SV, Wotton CJ, Handel AE, Yeates D, Goldacre MJ. Risk of venous thromboembolism in people admitted to hospital with selected immune-mediated diseases: record-linkage study. BMC Med 2011;9:1.

6. Matta F, Singala R, Yaekoub A, Najjar R, Stein P. Risk of venous thromboembolism with rheumatoid arthritis. Thromb Haemost 2009; 101:134-8.

7. Pincus T, Brooks RH, Callahan LF. Prediction of long-term mortality in patients with rheumatoid arthritis according to simple questionnaire and joint count measures. Ann Intern Med 1994;120:26-34.

First Release November 1 2019; J Rheumatol 2020;47:1; doi:10.3899/jrheum.190899 